\title{
XII.
}

Aus der Nervenklinik der königl. ungar. Universität in Budapest (Direktor: Prof. E. Jendrássik).

\section{Augenmuskellähmungen in Folge chronischer Blei- und Nikotinvergiftung.}

\author{
von

\section{Dr. Dezsö Hammer.}

I.

L. M., 16jährige Porzellanfabriksarbeiterin, kam in das klin ische Ambulatorium mit der Klage, dass sie an starken Bauchschmerzen, Verstopfung und Sehbeschwerden leidet.

Die Anamnese wies folgende Daten auf:

Thre Grosseltern sind in hohem Alter an unbekannten Krankheiten gestorben; ihr Vater ist 37, ihre Mutter 35 Jahre alt, beide sind-gesund; fünf jüngere Geschwister der Kranken sind ebenfalls gesund. Unsere Kranke litt in ihrer Kindheit an Rhachitis, doch lernte sie gehen und sprechen zu rechter Zeit. Seit ihrer Kindheit war sie, bis zum Beginne ihrer jetzigen Krankheit, stets gesund; seit August 1901 arbeitet sie in einer Porzellanfabrik, wo sie die Glasur zum Uberziehen der Porzellangegenstände bereitet. Während der Bereitung dieser Glasur steigen Stäubchen in die Luft; ausserdem können die Arbeiter ihre Hände auch für die Dauer der Mahlzeit von diesem Staube nicht vollkommen rein waschen.

Bereits einen Monat nach ihrem Eintritt in diese Fabrik fing sie an zu krănkeln; ihr Gesicht wurde bleich; sie verlor ihren früheren guten Appetit; sie erbrach von Zeit zu Zeit, bekam oft Bauchschmerzen und Verstopfung, welche sogar 3-4 Tage anhielten. Dieser Zustand verschlimmerte sich später; die Schmerzen quälten sie hauptsächlich in der Nabelgegend, von wo sie nach abwärts ausstrahiten. Manchmal hatte sie auch wochenlang andauernde sehr heftige reissende Kopfschmerzen; auch die Ohren schmerzten sie; seit Januar 1902 hat sich auch ihr Sehvermögen verschlechtert. Dieses letztere Übel führte sie zum Herrn Privatdozenten Dr. Mohr, der die Kranke mit dem Augenbefunde zur Behandlung auf unsere Klinik schickte.

Bei ihrer Aufnahme fanden wir folgendes: Patientin ist ein ziemlich gut entwickeltes Mädchen von blasser Hautfarbe; Schleimbäute sind bleich, am Halse sind zahlreiche linsen- und erbsengrosse Drüsen fühlbar; die Schilddrüse ist gut fühlbar; auf den Zähnen ist ein schmaler, aber gut erkennbarer graner Bleisaum sichtbar; die Zunge ist belegt. Der Bauch 
ist eingezogen, bei Druck etwas empfindlich; Stuhl träge, nur nach Gebrauch von Abführmitteln; Urin und Urinentleerung sind normal.

Beide Pupillen sind gleich, mittelweit, sie reagieren gegen Licht und Accomodation gut; die Refraktion ist normal; die Papillen sind geschwollen, ihre Ränder verschwommen; die Gefässe stellenweise mit Exsudat bedeckt; die Venen geschlängelt, mit punktförmiger Blutung; die Adern der Retina sind auch anderswo mit Exsudat bedeckt (Papilloretinitis).

Die Sehschärfe ist auf beiden Augen $5 / 20$; ausserdem ist beiderseits der Nervus abducens gelähmt; ferner ist die Bewegung der Augen nach aufwärts auch stark erschwert; es besteht somit Strabismus convergens.

Der Patellarsehnenreflex ist auf der rechten Seite auslösbar, auf der linken jedoch nur durch den Jendrássikschen Handgriff; die Achillessehnenreflexe hingegen sind auch auf diese Weise nicht auszulösen.

Therapie: Jodkalium, Bäder.

Zwei Wochen später haben wir folgendes aufgezeichnet: Die Achillessehnenreflexe sind auf beiden Seiten schwach, die Hautreflexe sind normal.

Nach-weiteren zwei Wochen haben die Bauchschmerzen aufgehört. Die Patellar- und Achillessehnenreflexe konnten schon leichter ausgelöst werden. Die Sehschärfe war auf dem rechten Ange $5 / 10$, auf dem linken $5 / 7$; am Augenhintergrunde bestand noch die hochgradige Papilloretinitis; auch gelbe Flecken waren zu sehen (Dr. Kornel Scholtz).

Der Zustand der Patientin besserte sich auch weiterhin; sechs Wochen nach der Aufnahme war das Doppeltsehen nicht mehr vorhanden; der Visus hat sich wieder gebessert und betrug auf der rechten Seite $5 / \pi$, auf der linken $5 / 5$, das Bild des Augenhintergrundes hat sich nicht wesentlich verändert. Die Kranke verliess bald darauf, funktionell geheilt, die Klinik, hat einen anderen Lebensunterhalt gefunden und, wie ich späterhin noch erfuhr, hält sie sich für vollkommen gesund. Leider konnte keine Augenspiegeluntersuchung ausgeführt werden.

Aus dem Krankheitsverlauf dieses Falles sehen wir, dass die Patientin äusserst empfindlich für Blei war, indem schon nach einem Monat der Beschäftigung in der Fabrik sich die ersten Symptome der Bleivergiftung zeigten; trotzdem hat sie acht Monate noch in derselben Porzellanfabrik gearbeitet, ohne die Vorsichtsmassregeln streng zu beobachten. Die schweren Vergiftungserscheinungen zeigten sich zunächst in zeitweisen Kopfschmerzen, später zeigte sich öfters Brechreiz und Erbrechen, erst dann begannen die Augenstörungen.

Als interessante Erscheinung müssen wir das vorübergehende Fehlen der Sehnenreflexe noch betonen. Schon bei der ersten Untersuchung waren beide Achillessehnenreflexe nicht auslösbar und der linksseitige Patellarreflex auch nur durch den Jendrássikschen Handgriff; ja im Laufe der Beobachtung war der rechtsseitige Patellarsebnenreflex auch einige Zeit vollkommen fehlend. Im weiteren Verlauf der Behandlung kamen nach und nach die Sehnenreflexe wieder zum Vorschein, und als Patientin von der Klinik entlassen wurde, waren 
sowohl die Patellar- als auch die Achillessehnenreflexe schon auf beiden Seiten gleich lebhaft auslösbar.

Auf Grund der aufgezählten Symptome ist die Entstehungsursache des Krankheitsbildes ganz unzweifelhaft. Der gleichzeitige Eintritt und besonders die prompte Genesung der Augenstörungen mit den übrigen Erscheinungen der Bleivergiftung beweist die Zusammengehörigkeit des ganzen Symptomenkomplexes.

Wenn wir die Literatur der Bleivergiftung durchmustern, finden wir keinen Fall, der dem unserigen in allem ähnlich wäre; doch sind demselben nahestehende Fälle bereits, wenn auch in geringer Anzahl, aufgezeichnet. So finden wir Berichte über Fälle, in welchen, in Verbindung mit der Bleivergiftung, Augenmuskellähmungen vorgekommen sind. In dieser Beziehung am ähnlichsten dem unserigen ist ein Fall von Schröder ${ }^{1}$ ); in diesem waren auf beiden Augen Abducens-Paresen und Neuroretinitis vorhanden mit geringen Blutungen im Augenhintergrunde. In diesem Fall waren klinisch Strabismus convergens, Doppeltsehen vorhanden, die später geheilt sind; neben diesen Erscheinungen bestand auch Lähmung des N. radialis.

Zinken ${ }^{2}$ ) erwähnt eine linksseitige Abducenslähmung mit Strabismus convergens oculi dextri und Diplopia, nebst den allgemeinen Symptomen der Bleivergiftung. Im Falle von Stood ${ }^{3}$ ) entstanden Kopfschmerzen, Delirium, chronische Krämpfe, linksseitige Abducenslähmung und Strabismus convergens, später Atrophie des Sehnerven. Mayer ${ }^{4}$ ) erwähnt in einem Falle eine beiderseitige Parese des Abducens und Neuritis nervi optici. Galezovsky ${ }^{5}$ ) beschreibt in einem Falle eine plötzlich eingetretene, aber nicht vollständige beiderseitige Abducenslähmung und eine linksseitige Oculomotoriuslähmung; in einem zweiten Falle waren alle äusseren Augenmuskeln völlig gelähmt und auch die Accomodation war gestört. Derselbe beobachtete in einer chronischen Bleivergiftung auch noch Mydriasis und Paresis accomodationis, aber ohne Störung der Augenmuskeln. Landesberg ${ }^{6}$ ) sah in einem Falle, ausser den gewöhnlichen Symptomen der Bleivergiftung, eine später geheilte rechtsseitige Paresis abducentis; in einem anderen Falle aber, bei völliger Lähmung des äusseren Zweiges des rechtsseitigen Oculomotorius (mit Ptosis) auf derselben Seite Myosis. Der Kranke von Wadsvorth ${ }^{7}$ ) konnte das linksseitige Auge nach ein- und auswärts, sowie nach aufwärts gut bewegen; aber beim Abwärtssehen war die Bewegung beschränkt; hingegen ist die Bewegung des rechten Auges nur nach aufwärts gehemmt gewesen.' Am Augenhintergrunde war das Bild der Neuritis optica zu sehen. Sauvinea ${ }^{8}$ ) hat sämtliche Bewegungen der Augen auf beiden Seiten beschränkt gefunden; es bestand, nebst guten Pupillarreflexen, auf 
beiden Augen eine völlige Ophthalmoplegia externa; die Sehnenreflexe waren abgeschwächt. Mannaberg ${ }^{9}$ ) beschrieb eine rechtsseitige Oculomotoriuslähmung mit totaler rechtsseitiger peripherischer Facialislähmung und mit Neuritis optica. Lantsheere-Blyckaerts ${ }^{10}$ ) erwähnt in einem Falle Strabismus divergens, Augenmuskelschwäche; in einem anderen eine linksseitige Lähmung des Abducens, Pupillendifferenz, Accomodations-Abschwächung und Atr. n. optici; im dritten Falle ist nur die Convergenz mangelhaft gewesen. Gayet ${ }^{11}$ ) beschrieb eine rechtsseitige Ptosis, eine Oculomotoriuslähmung, Mydriasis und Accomodationsstörung, Bei dem Kranken von Aurand ${ }^{12}$ ) waren die inneren geraden Augenmuskel gelähmt und die Accomodation geschwächt; in einem Falle spielt aber auch Alkoholismus und Arteriosklerose eine Rolle. Focker ${ }^{13}$ ) erwähnt nur eine Diplopie. In der Krankengeschichte, mitgeteilt von Vulpian und Raymond ${ }^{14}$ ), finden wir nur eine linksseitige erweiterte Pupille und Ataxie. Meyer ${ }^{15}$ ) beobachtete bei einem 19jährigen Mädchen, einer Brüsseler Spitzenklöpplerin, im Verlaufe der Bleivergiftung ausser Kolik Hemiplegie, Strabismus und Diplopie, welche nach 6 Monaten verschwanden; indessen entstand späterhin auf beiden Augen der Kranken Atrophia nerv. optici. Lann ${ }^{16}$ ) beschreibt einen Fall von Saturninvergiftung mit Diplopie; in diesem Falle entwickelte sich später, nach einem heftigen Kopfschmerz, Atrophie des N. opticus. Stood ${ }^{17}$ ) führt in seinem schon oben erwähnten Werke noch zwei Fälle an, in deren einem nur eine Accomodationsschwäche bestand, im anderen - nebst der stärkeren Accomodationsstörung - - noch geschwollene und hyperämische Papillen vorhanden waren. Frank ${ }^{18}$ ) macht in der Beschreibung eines sehr schweren Krankheitsfalles Erwähnung von den sehr erweiterten Pupillen, welche selbst auf das stärkste Licht nicht reagierten. In dem Falle von $W_{\text {ood }}{ }^{19}$ ) war - ausser Oculomotoriuslähmung, linksseitiger Ptosis und ausser der absoluten Pupillenstarre sowohl gegen das Licht als auch bei der Accomodation - kein Symptom der Bleivergiftung vorhanden gewesen. $\mathrm{Bach}{ }^{20}$ ) beschreibt einen Fall, in welchem auf der rechten Seite eine vollkommene Oculomotoriuslähmung, auf der linken Paresis des M. rect. lateralis und des M. obl. superior aufgezeichnet sind, und die Accomodation auf der linken Seite völlig gelähmt und auch auf der rechten Seite stark geschwächt war. Bach hält diesen Fall für Bleivergiftung; indessen kanin man hier Tabes nicht gänzlich ausschliessen. Bei dem Kranken Lagleyrés ${ }^{21}$ ) ist von den Augenmuskeln nur der rechtsseitige M. rect. medialis gelähmt gewesen. Janovsky ${ }^{22}$ ) erwähnt eine engere linke Pupille, die gegen Licht nur träge reagierte, ausserdem war der untere Zweig des $\mathbf{N}$. facialis teilweise gelähmt. In dem durch Blei- 
vergiftung entstandenen Fall von Hemiatrophia linguae, mitgeteilt von Remak ${ }^{23}$ ), waren auf dem linken Auge schwache Ptosis und reflektorische Pupillenstarre vorhanden. Sachs ${ }^{24}$ ) beschreibt Spasmus des M. rect. medialis in einem Bleivergiftungsfalle.

Dies sind die Fälle, welche ich in der Literatur verzeichnet fand. Wir sehen aus diesen, dass die Augenmuskellähmungen im Zusammenhange mit der Bleivergiftung verhältnismässig in nicht vielen Fällen vorkommen; insbesondere aber sind die Abducensläbmungen selten. Beiderseitige Paresis abducentis kam bloss in drei Fällen vor; in den übrigen Fällen war eine einseitige Lähmung des Abducens nebst Lähmung der in den Bereich anderer Nerven gehörigen Muskeln vorhanden. Bezüglich des an unserer Kranken bestandenen vorübergehenden Fehlens der Sehnenreflexe, welches Symptom an den sonst vollkommen gesunden unteren Extremitäten zu beobachten war und im Laufe der Behandlung gänzlich geheilt wurde, fand ich in der Literatur der Bleivergiftung kein Beispiel. Es kommen zwar in mehreren von den aufgeführten Fällen Erwähnungen über die Sehnenreflexe vor, die entweder verschwunden oder lebhaft gewesen sind, aber immer nur in solchen Fällen, in welchen im Kreis der krankhaften. Symptome auch die Muskeln der unteren Extremitäten teilnahmen; folglich können wir in diesen Fällen die Verletzung eines Teiles der Reflexbahn annehmen; in unserem Falle aber, wie dies die Krankheitsgeschichte beweist, hat die Bleivergiftung — das Auge ausgenommen - nirgends im ganzen Organismus, in der Muskulatur noch weniger in der Sensibilität andere Symptome verursacht.

Der schon seit langer Zeit bestehende Streit über die Frage, ob die durch die Bleivergiftung verursachten Lähmungen von der Läsion der zentralen Nervenelemente, von der Intoxikation der peripherischen Nervenfasern, oder von der Läsion der Muskeln herrühren, ist noch lange nicht entschieden. Durch die jetzigen Untersuchungsmittel ist das Studium dieser Frage so gut wie erschöpft und die endgültige Entscheidung wird wahrscheinlich nur mit Hilfe neuer Methoden möglich werden. Vorläufig müssen wir uns damit begnügen, weṇn wir zur Vervollständigung des klinischen Krankheitsbildes mitwirken können.

II.

Augenmuskellähmungen durch Nikotinvergiftung kommen sehr selten vor. Nur zwei Fälle konnte ich in der Literatur finden; den einen teilt Fontan mit, der andere ist der Fall Jans, den Dufour erwähnt. 
In dem Falle von Fontan ${ }^{25}$ ) ist beiderseitige Oculomotoriuslähmung vorhanden gewesen. Auf der rechten Seite war der M. rect. medialis gelähmt, auf der linken sämtliche äussere Augenmuskeln. Die Pupillen waren erweitert. $\nabla$ on den allgemeinen Symptomen der Vergiftung waren notiert: starke Kopfschmerzen, Schwindel, Schlaflosigkeit und Störungen in der Ernährung. Die Augenmuskellähmung ist plötzlich entstanden und auf Abstinenz hat sie sich gebessert.

In dem Falle $\mathrm{Jans}^{26}$ ) ist gleichfalls eine Oculomotoriuslähmung vorhanden gewesen, welche sich während 4 Wochen auf Abstinenz gleichfalls gebessert hat.

Ausser diesen erwähnt Eperon, dass or in einem Nikotinvergiftungsfalle auf Tabes hinweisende Symptome beobachtete und zwar: das Fehlen des Patellarreflexes, lanzinierende Schmerzen, einmal Rombergsches Symptom, in drei Fällen reflektorische Pupillenstarre. Es ist schwer, die Tabes in solchen Fällen auszuschliessen.

Unseren Fall teile ich in folgendem mit:

W. L., 59 Jahre alt, Hausierer, erschien am 4. Nov. 1902 in dem klinischen Ambulatorium. Aus der Anamnese des Kranken hebe ich hervor, dass er im 24. Lebensjahre heiratete und 2 gesunde Kinder hat; im 47. Lebensjahre hat er ein Ulcus acquiriert and infolge sekundärer Symptome Einreibungen mit grauer Salbe gebraucht. Bezüglich seiner Lebensweise machte er die Angabe, dass er ein starker Raucher gewesen ist; er hat täglich 8-9, hie und da anch 15 starke Zigarren geraucht.

Mitte September 1902 bemerkte er, dass er doppelt sieht, auch ungefähr seit dieser Zeit sieht er trübe und empfand zuweilen in den Augen geringfügige Schmerzen.

Wir notierten bei der Aufnahme: Der linksseitige Nervus abducens und Nerv. oculomotorius waren gelähmt; am rechten Auge war eine leichte Ptosis zu bemerken. Die Pupillen waren gleich; sie reagierten gegen Licht gar nicht, gegen Accomodation kaum. Laut Befund der auf der Augenklinik durchgeführten genauen Untersuchung war die Sehschärfe auf dem rechten Auge $5 / 70$, auf dem linken 5/50, nebenbei ist ein zentrales Skotom für Rot und Grün festgestellt worden. Die oculistische Diagnose hat als Ursache des Schlechtsehens Neuritis retrobulbaris toxica bezeichnet (Herr Assistent Dr. Blaskovits).

Was die Lähmung der Muskeln anbelangt, so haben wir die Bewegung des linken Augapfels nach aufwärts, auswärts und einwärts stark beschränkt gefunden, respektive der M. rect. sup., der M. rect. lateralis und M. rect. medialis waren schwach; auch auf der rechten Seite ist der M. rect. sup. schwach, mithin bestand Parese der beiderseitigen Nn. oculomotorii und Lähmung des linksseitigen N. abducens.

Chronischer Rachenkatarrh. Herz nicht vergrössert, die Herzaltion unregelmässig, arythmisch, Herztöne rein. Subjektiv Klagen über Schwindel.

Über Schmerzen klagte der Kranke nicht, Rombergsches Symptom und Hypotonie waren nicht vorhanden, im Tastgefühl war es nicht mög- 
lich Fehler zu finden: die Patellar- und Achillessehnenreflexe waren gut auslösbar, ja sie waren sogar ein wenig gesteigert; die inneren Organe waren gesund; Urinentleerung normal; im Urin waren keine fremden Bestandteile; der Appetit ist gut; Stuhl regelmässig.

Die Ergebnisse dieser Untersuchungen betrachtend, sehen wir, dass ein auf Tabes hinweisendes Symptom in unserem Falle nicht vorhanden war. Bei der tabischen Erblindung bleibt das zentrale Sehvermögen gewöhnlich lange Zeit intakt, denn die Veränderungen offenbaren sich anfänglich in der Einschränkung des peripherischen Gesichtsfeldes und erst von da ab verbreitet sich die Abnahme der Sehschärfe gegen das Zentrum. Hier ist aber gerade das zentrale Sehvermögen zugrunde gegangen. Die durch die Augenuntersuchung festgestellte Neuritis retrobulbaris ist gleichfalls nicht tabischen Ursprunges, sondern beweist die Entstehung durch Vergiftung.

In unserem Falle sind also einesteils das vollständige Fehlen tabischer Symptome, andererseits aber die Bedingung und die klinische Krankheitsform der Nikotinvergiftung: die Neuritis retrobulbaris und das zentrale Skotom, vorhanden. Unter solchen Umständen scheint es berechtigt, auch die Augenmuskellähmung als ein mit der Sehstörung gleichzeitig und aus derselben. Ursache entstandenes Krankheitssymptom zu betrachten.

Dem Patienten wurde das Rauchen strengstens untersagt, doch besserten sich die Augensymptome nicht.

\section{Literatur.}

1) Schröder, Arch. f. Ophthalm. 1885. Bd. 31. 1. S. 229.

2) Zinken ref. Arch. f. Ophth. Bd. 31. 1. 238.

3) Stood, Gräfes Arch. Bd. 30. 3. 215.

4) Mayer, Jahrbuch f. Psych. 1891. 10. S. 69.

o) Galezorsky, Recueil d'opht. 187\%. p. 264.

6) Land esberg, Med. Bull. Philad. 2. p. 108. 1880.

7) Wadsvorth, Boston Med. and Surgic. Journ. 1885.

8) Saurineau, Thèse de Paris 1892.

9) Mannaberg, Recueil d'opht. 1897.

10) Lantsheere-Blyckaerts, Recueil d'opht. 1900.

11) Gayet, Aurands Fall beschreibt Bennet, Contribution à l'Étude des Paralyses ocrlaires d'origine toxique. Lyon 1902.

12) Dasselbe.

13) Focker, Annales d'oculistique 1898. 
14) Vulpian und Raymond, Thèse de Renaut.

15) Meyer, L'Union. Med. 1868. Nr. 78.

16) Lun n, Medical Times 1872.

17) Stood, Gräfes Archiv. Bd. 30. 3. 21 .

18) Frank, Wien. med. Presse 1883. 698.

19) Wood, Med. News 1897. 70. 23. p. 700.

20) Bach, Archiv f. Augenheilkde. 1893. 26. S. 218.

21) Lagleyré, Clinique opht. Nr. 8. 1896.

22) Janovsky, Neurol. Zentralbl. 1895.

23) Remak, Berl. klin. Wochenschr. 1886.

24) Sachs, Archiv f. Augenheilkde. 1898, 37. 1. S. 9.

25) Fontan, Recueil d'opht. 1883. 309.

26) Marina, Über multiple Augenmuskellähmungen. Leipzig 1896. 